\title{
Growth Behavior
}

National Cancer Institute

\section{Source}

National Cancer Institute. Growth Behavior. NCI Thesaurus. Code C123892.

An increase in size or volume of cells in culture. 